\title{
Healthcare resource utilization and costs associated with inflammatory bowel disease among patients with chronic inflammatory diseases: a retrospective cohort study
}

David P. Hudesman ${ }^{1 *}$, Soumya D. Chakravarty ${ }^{2,3^{*}}$, Bruno Emond ${ }^{4}$, Lorie A. Ellis ${ }^{2}$, Patrick Lefebvre ${ }^{4}$, Kay Sadik ${ }^{2}$ and Jose U. Scher ${ }^{1}$

\begin{abstract}
Background: Chronic inflammatory diseases (CIDs; ankylosing spondylitis [AS], psoriatic arthritis [PsA], psoriasis [PsO], or rheumatoid arthritis [RA]) and inflammatory bowel disease (IBD; Crohn's disease and ulcerative colitis) are associated with substantial economic burden. The relative increased costs among patients with CIDs and concomitant IBD compared to those without IBD is an important consideration when deciding on the clinical management of patient symptoms. Given the increasing use of novel agents for the treatment of CIDs, including those that may increase the risk of IBD in patients with CIDs, the objective of the study was to describe the incidence of IBD and to quantify healthcare resource utilization (HRU) and costs associated with IBD among patients with CIDs.
\end{abstract}

Methods: The IBM MarketScan ${ }^{\circledR}$ Research Databases (1/2010-7/2017) were used to identify adult patients with $\geq 2$ claims with a diagnosis of either AS/PsA/PsO/RA (index date was a random claim for AS/PsA/PsO/RA). The one-year incidence rate of IBD was calculated following the index date. HRU and healthcare costs were compared between patients developing and not developing IBD in the year following the index date, adjusting for baseline characteristics.

Results: A total of 537,450 patients with CIDs (mean age $=54.0$ years; $63.1 \%$ female) were included in the study. The 1-year incidence rate of IBD was $0.52 \%$ (range $=0.39 \%$ in patients with PsO but without PsA to $1.73 \%$ in patients with AS). Patients who developed IBD $(N=2778)$ had significantly higher rates of inpatient, outpatient, and emergency room visits (incidence rate ratios $[\mathrm{IRR}]=2.91,1.35,1.81$; all $P<0.0001$ ), compared to patients without IBD $(N=534,672)$. Patients who developed IBD had $\$ 18,500(P<0.0001)$ higher total costs per year, including $\$ 15,121$ $(P<0.0001)$ higher medical costs and $\$ 3380$ higher pharmacy costs $(P<0.0001)$.

Conclusion: Higher HRU and costs were observed in patients with concomitant CID and IBD compared to patients with CID alone. Consideration should be given to treatment decisions that adequately manage CID and IBD to ensure optimal clinical and economic outcomes.

Keywords: Inflammatory bowel disease, Crohn's disease, Ulcerative colitis, Psoriasis, Psoriatic arthritis, Ankylosing spondylitis, Rheumatoid arthritis, Healthcare costs, Healthcare resource utilization

\footnotetext{
* Correspondence: David.Hudesman@nyulangone.org; schakr66@ITS.JNJ.com ${ }^{1} \mathrm{NYU}$ Langone Health, 240 East 38th Street, 23rd Floor, New York, NY 10016, USA

2Janssen Scientific Affairs, LLC, 800 Ridgeview Drive, Horsham, PA 19044, USA

Full list of author information is available at the end of the article
}

(c) The Author(s). 2020 Open Access This article is distributed under the terms of the Creative Commons Attribution 4.0 International License (http://creativecommons.org/licenses/by/4.0/), which permits unrestricted use, distribution, and reproduction in any medium, provided you give appropriate credit to the original author(s) and the source, provide a link to the Creative Commons license, and indicate if changes were made. The Creative Commons Public Domain Dedication waiver (http://creativecommons.org/publicdomain/zero/1.0/) applies to the data made available in this article, unless otherwise stated. 


\section{Background}

Inflammatory bowel disease (IBD; Crohn's disease and ulcerative colitis) is characterized by chronic inflammation of the gastrointestinal tract and is a common extraarticular manifestation in patients with chronic inflammatory diseases (CIDs), such as ankylosing spondylitis (AS), psoriatic arthritis (PsA), psoriasis (PsO), and to a lesser extent in patients with rheumatoid arthritis (RA) [1-4]. Distinct CIDs and IBD share similar genetic susceptibility loci and pathological mechanisms including multiple inflammatory cytokines and immune-signaling pathways [5-10].

Given the pathophysiological relationships between CID and IBD, many pharmacological treatments target manifestations of both syndromes [11-13]. These include disease-modifying anti-rheumatic drugs (DMARDs), corticosteroids, and biologic therapies. However, there is evidence suggesting that some of these treatments may increase the risk of developing new onset IBD or exacerbating existing IBD in patients with CIDs $[12,14,15]$. The use of certain biologic therapies such as etanercept (a tumor necrosis factor inhibitor) and interleukin-17 (IL-17) antagonists in patients with CIDs is cautioned due to possible increased risk of IBD [16-22].

While multiple previous studies have assessed the incremental burden of IBD relative to controls without IBD [23-27], little is known on the burden of IBD in patients with pre-existing CID. A recent real-world analysis of the economic burden associated with IBD among patients with PsA and AS in the United States (US) showed that compared to patients without IBD, those with IBD had significantly higher total healthcare costs-up to $27 \%$ higher in patients with PsA and $38 \%$ higher among patients with AS [28]. However, this analysis exclusively focused on patients with PsA or AS; thus, it is unclear whether the conclusions of this study can be generalized to other patients with CID.

The objectives of the current study were to describe the incidence of IBD among patients with CIDs and to compare HRU and costs among patients with CIDs who developed IBD versus patients with CIDs who did not develop IBD.

\section{Methods}

\section{Data source}

This analysis utilized data from the IBM MarketScan ${ }^{\oplus}$ Research Databases (01/01/2010-07/31/2017), which include the Commercial Claims and Encounters database and the Medicare Supplemental and Coordination of Benefits database. The databases comprise claims from approximately 75 million individuals covered by 100 payers. The databases cover all census regions of the US and provide eligibility-related information as well as outpatient medical claims, inpatient medical claims, and outpatient drug dispensing claims information. Data were de-identified and the databases were fully compliant with the Health Insurance Portability and Accountability Act and thus, no ethics board review was required.

\section{Study design}

A retrospective longitudinal cohort study design was used. The index date for patients with AS, PsA, PsO, or RA was randomly assigned among service dates with a claim related to these CIDs. The rationale was to capture a prevalent population of patients with CIDs and to minimize the potential bias associated with the selection of the most or least recent dates associated with such claims, which could lead to the inclusion of patients with new-onset or advanced CID. When evaluating the incidence of IBD, a non-CID population was also studied to put in perspective the incidence of IBD in patients with CIDs to that of the general population. For the non-CID population, the index date was randomly assigned among all dates for which a service was provided. The baseline period was defined as the 12-month period prior to the index date. The observation period was defined as the 12-month period following the index date (i.e., patients were censored 12 months after the index date; Fig. 1).

\section{Study sample}

To be included in the study sample, patients were required to be $\geq 18$ years of age as of the index date and have $\geq 12$ months of continuous health plan enrollment pre- and post-index date. Patients in the CID cohort were required to have $\geq 2$ claims with the same diagnosis among the following CIDs: AS (International Classification of Diseases, Ninth Revision, Clinical Modification [ICD-9 CM] code 720.0; International Classification of Diseases, Tenth Revision, Clinical Modification [ICD-10 CM] code M45.x), PsA (ICD-9 CM code 696.0; ICD-10 CM code L40.5x), PsO (ICD-9 CM code 696.1; ICD-10 CM codes L40.0-L40.4, L40.8, or L40.9), or RA (ICD-9 CM code 714.0; ICD-10 CM codes M05.1-M05.9, or M06). Patients with $\geq 2$ claims with a diagnosis of IBD (i.e., Crohn's disease [ICD-9 CM code 555.x; ICD-10 CM code K50.x] or ulcerative colitis [ICD-9 CM code 556.x; ICD-10 CM code K51.x]) during the 12-month baseline period were excluded. Moreover, patients with cancer treated with rituximab or ofatumumab during the baseline period were excluded because it could not be determined using claims data whether these agents were prescribed to treat cancer or CID. Patients with a transplant procedure were also excluded because of the high costs usually associated with this procedure, which may skew results. The non-CID cohort was composed of individuals with no claim with a diagnosis of $\mathrm{AS}, \mathrm{PsA}, \mathrm{PsO}$, or RA at any 


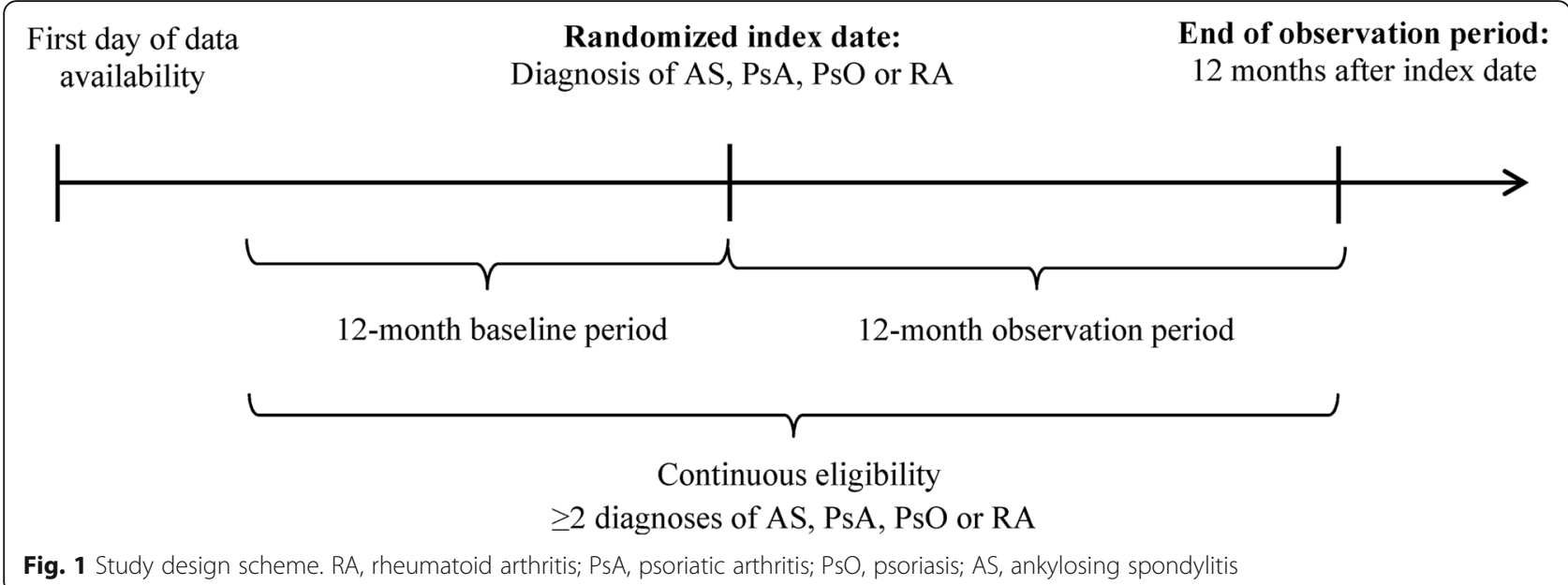

time. Similar to the CID cohort, patients in the nonCID cohort were additionally required to be $\geq 18$ years of age as of the index date and have $\geq 12$ months of continuous health plan enrollment pre- and postindex date. Patients with cancer treated with rituximab or ofatumumab, with a transplant procedure, or with $\geq 2$ claims with a diagnosis of IBD during the 12-month baseline period were excluded.

\section{Study cohorts}

Patients with CIDs were classified into six cohorts: (1) all patients with CIDs (i.e., AS, PsA, PsO, or RA); (2) patients with RA; (3) patients with PsO and PsA; (4) patients with PsO but without PsA; (5) patients with AS; and (6) patients with AS, PsA, or PsO. Eligible patients with claims for more than one CID were classified into each eligible CID cohort (i.e., one patient could be present in more than one cohort). Each of the six cohorts mentioned above was analyzed separately.

\section{Study measurements}

Baseline characteristics included age, gender, type of insurance plan, region of residence, year of index date, Quan-Charlson Comorbidity Index (Quan-CCI), extraarticular manifestations (EAMs), gastro-related conditions, drug use, all-cause HRU, and all-cause costs.

Patients developing IBD were identified by the presence of $\geq 2$ claims with a diagnosis of IBD during the course of the observation period. The one-year incidence rate (IR) of IBD was calculated for each cohort. The numerator was defined as the number of patients with $\geq 2$ claims with an IBD diagnosis during the period of evaluation (i.e., 1 year), and the denominator was defined as the total number of patients in the cohort studied. As a sensitivity analysis, the oneyear prevalence of IBD among patients with or without a prior IBD diagnosis was also reported for each cohort. The numerator and denominator definitions used were the same as the ones used to measure incidence, except that patients who had a prior IBD diagnosis were not excluded from the numerator and denominator.

HRU outcomes included number of inpatient admissions, number of days of inpatient stay, number of days with outpatient services, number of days with emergency room (ER) visits, and number of days with durable medical equipment use. The number of days with a surgery was also evaluated.

All-cause total healthcare costs were stratified by medical and pharmacy costs. Medical costs were further stratified by inpatient, outpatient, ER, and durable medical equipment costs. Costs related to surgery were also evaluated. By definition, all-cause healthcare costs included any healthcare costs incurred during the followup period, regardless of whether they were related to CIDs, IBD (e.g., endoscopies, colonoscopies), or other comorbidities.

\section{Statistical analysis}

Baseline characteristics were summarized using means and standard deviations (SDs) for continuous variables and proportions for categorical variables. The oneyear IR of IBD was reported as a proportion of patients.

The rates of HRU between patients with and without IBD among the six cohorts were compared using generalized linear models (GLMs) with a Poisson distribution adjusting for age, gender, region, type of insurance plan, Quan-CCI, and type of CID (i.e., RA, $\mathrm{PsA}, \mathrm{PsO}$, or AS); incidence rate ratios (IRRs) with corresponding 95\% confidence intervals (CIs) and $p$ values were reported. GLMs with normal distribution were used to compare costs between patients with 
and without IBD among the six cohorts, adjusting for age, gender, region, type of insurance plan, Quan-CCI score (a score derived from a number of comorbidities [e.g., hypertension, diabetes, chronic pulmonary disease] and their associated risk of inhospital mortality [29]), and type of CID (i.e., AS, PsA, PsO, or AS); mean yearly cost differences (MYCDs) and the corresponding 95\% CIs and $p$ values were reported. Non-parametric bootstrap procedures were used to evaluate statistical significance and 95\% CIs. In a sensitivity analysis, costs were evaluated in the subset of patients with AS, RA, PsA, or PsO who received DMARDs and/or corticosteroids.

Cost and HRU outcomes were evaluated per-patientper-year (PPPY). Cost outcomes were inflated to 2017 US dollars (USD) using the medical component of the US Consumer Price Index.

\section{Results}

\section{Baseline characteristics}

A total of 537,450 patients with CIDs, including 206,260 patients with RA; 21,250 patients with $\mathrm{PsO}$ and PsA; 124,950 patients with $\mathrm{PsO}$ but without PsA; 16,029 patients with AS; and 183,318 with AS, PsO, or PsA composed the study's analytical sample (Fig. 2). Between the RA, PsO with PsA, PsO without PsA, and AS patient cohorts, 3280 patients were both in the RA and PsO with PsA cohorts, 3310 patients were both in the RA and $\mathrm{PsO}$ without PsA cohorts, 2957 patients were both in the RA and AS cohorts, 276 patients were both in the $\mathrm{PsO}$ with PsA and AS cohorts, and 288 patients were both in the PsO without PsA and AS cohorts. A total of $1,008,436$ patients were not diagnosed with CIDs.

In the CID and non-CID cohorts, mean age was 54.0 and 46.3 years, respectively; 63.1 and $55.1 \%$ were female. In the other condition-specific cohorts, mean age ranged from 49.4 years in the AS cohort to 57.5 years in the RA cohort, and the proportion of female patients ranged from $51.3 \%$ in the $\mathrm{AS}, \mathrm{PsO}$ or PsA cohort to $75.8 \%$ in the RA cohort. The most commonly used medications at baseline were corticosteroids $(45.3 \%)$, opioids (38.8\%), DMARDs (37.6\%) and non-steroidal anti-inflammatory drugs (NSAIDs; $33.3 \%)$. In the non-CID cohort, the most commonly used medications were opioids (24.0\%), corticosteroids (19.5\%), and NSAIDS (17.0\%). A higher proportion of patients in the RA cohort were using corticosteroids and DMARDs (62.3 and 67.1\%, respectively), and a higher proportion of patients in the $\mathrm{PsO}$ and PsA cohort were using biologics (54.3\%) compared to other cohorts (Table 1).

\section{One-year IR and prevalence of IBD}

The one-year IR and prevalence of IBD by type of CID are depicted in Fig. 3a and Fig. 3b, respectively. Among all patients with CIDs, the IR of IBD was $0.52 \%$. For patients in the AS cohort, the IR of IBD was numerically higher (1.73\%), compared to other CID cohorts (IR range: $0.39 \%$ in the $\mathrm{PsO}$ without PsA cohort to $0.54 \%$ in the AS, PsA, or $\mathrm{PsO}$ cohort) and the non-CID cohort (0.25\%). The prevalence of IBD was higher across all cohorts, but consistent trends were observed, with values ranging from $1.29 \%$ in patients with $\mathrm{PsO}$ without PsA to $6.05 \%$ in patients with AS. The prevalence of IBD was $0.60 \%$ in the non-CID cohort.

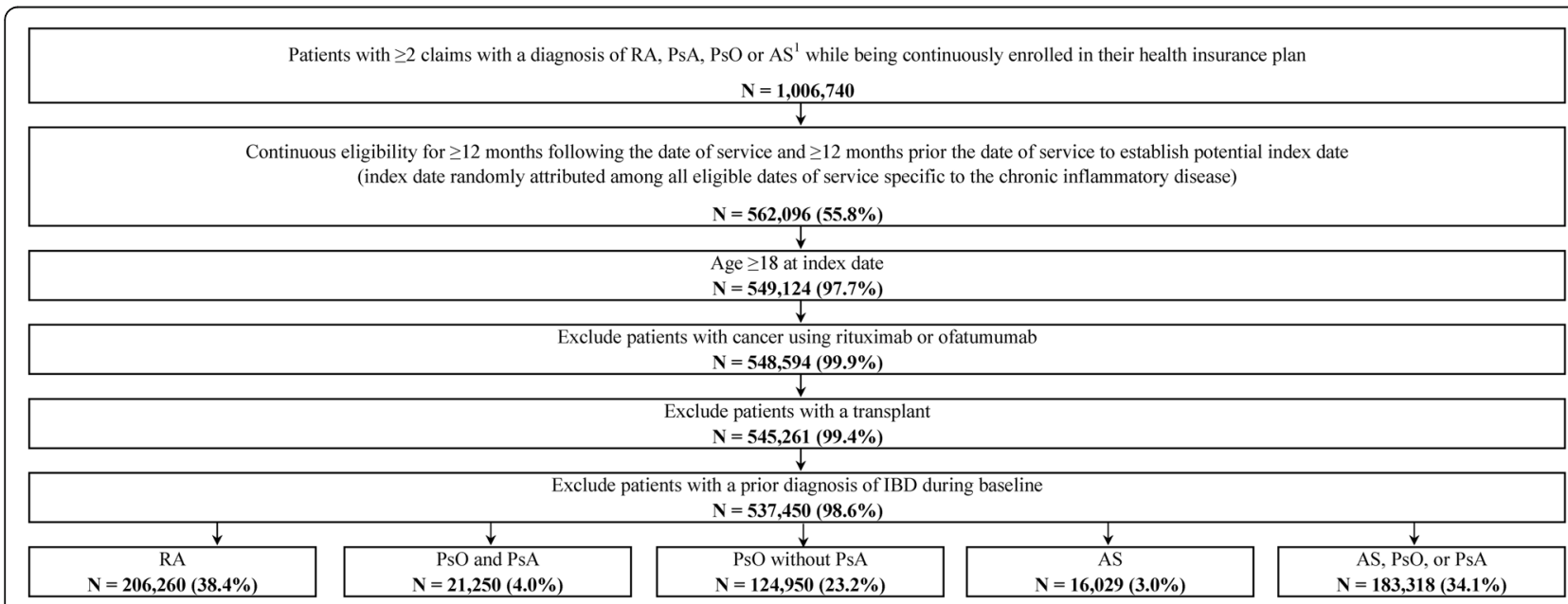

Fig. 2 Identification of the study population. RA, rheumatoid arthritis; PsA, psoriatic arthritis; PsO, psoriasis; AS, ankylosing spondylitis; IBD, inflammatory bowel disease. Notes: 1. Identified using the following ICD-9-CM and ICD-10-CM codes: RA (ICD-9-CM Code 714.0; ICD-10-CM code: M05.1-M05.9, M06), PsA (ICD-9-CM code 696.0; ICD-10-CM code: L40.5x), PsO (ICD-9-CM code 696.1; ICD-10-CM code: L40.0-L40.4, L40.8, L40.9), or AS (ICD-9-CM code 720.0; ICD-10-CM code: M45.x) 
Table 1 Demographic and clinical characteristics evaluated during the 12-month baseline period

\begin{tabular}{|c|c|c|c|c|c|c|c|}
\hline & $\begin{array}{l}\text { Non-CID cohort } \\
\text { (not diagnosed with } \\
\text { RA, PSA, PsO or AS) } \\
(N=1.008436 \text { ) }\end{array}$ & $\begin{array}{l}\text { All patients: RA, } \\
\text { PsO, PsA, or AS } \\
\text { cohort } \\
(N=537450)\end{array}$ & $\begin{array}{l}\text { Patients } \\
\text { with RA } \\
(N=206260)\end{array}$ & $\begin{array}{l}\text { Patients with } \\
\mathrm{PsO} \text { and PsA } \\
(\mathrm{N}=21250)\end{array}$ & $\begin{array}{l}\text { Patients with PsO } \\
\text { but without PsA } \\
(N=124950)\end{array}$ & $\begin{array}{l}\text { Patients } \\
\text { with AS } \\
(N=16.029)\end{array}$ & $\begin{array}{l}\text { Patients with } \\
\text { AS, PSO or PSA } \\
(N=183318)\end{array}$ \\
\hline$\overline{\mathrm{Age}^{\mathrm{a}}, \text { mean } \pm \mathrm{SD}}$ & $46.3 \pm 16.3$ & $54.0 \pm 14.7$ & $57.5 \pm 13.7$ & $51.4 \pm 12.2$ & $51.1 \pm 15.1$ & $494+138$ & $513+144$ \\
\hline Female, $\%$ & $55.1 \%$ & $63.1 \%$ & $75.8 \%$ & $52.8 \%$ & $51.5 \%$ & $43.8 \%$ & $51.3 \%$ \\
\hline \multicolumn{8}{|l|}{ Payment type, \% } \\
\hline Commercial & $88.9 \%$ & $79.3 \%$ & $73.6 \%$ & $89.0 \%$ & $83.6 \%$ & $88.3 \%$ & $84.9 \%$ \\
\hline Medicare & $11.1 \%$ & $20.7 \%$ & $26.4 \%$ & $11.0 \%$ & $16.4 \%$ & $11.7 \%$ & $15.1 \%$ \\
\hline \multicolumn{8}{|l|}{ Region of residence ${ }^{a}, \%$} \\
\hline South & $39.0 \%$ & $37.5 \%$ & $39.1 \%$ & $40.2 \%$ & $36.2 \%$ & $36.3 \%$ & $36.8 \%$ \\
\hline North Central & $21.9 \%$ & $22.4 \%$ & $23.0 \%$ & $20.2 \%$ & $22.4 \%$ & $19.1 \%$ & $21.7 \%$ \\
\hline West & $18.9 \%$ & $16.8 \%$ & $16.5 \%$ & $17.6 \%$ & $16.7 \%$ & $24.9 \%$ & $17.4 \%$ \\
\hline North East & $18.9 \%$ & $22.4 \%$ & $20.5 \%$ & $21.0 \%$ & $24.0 \%$ & $18.9 \%$ & $23.2 \%$ \\
\hline Unknown & $1.4 \%$ & $0.8 \%$ & $0.8 \%$ & $1.1 \%$ & $0.8 \%$ & $0.8 \%$ & $0.9 \%$ \\
\hline \multicolumn{8}{|l|}{ Year of index date, $\%$} \\
\hline 2011 & $30.9 \%$ & $24.8 \%$ & $28.2 \%$ & $23.4 \%$ & $26.0 \%$ & $25.7 \%$ & $25.9 \%$ \\
\hline 2012 & $19.2 \%$ & $17.8 \%$ & $17.5 \%$ & $14.4 \%$ & $17.8 \%$ & $16.6 \%$ & $17.4 \%$ \\
\hline 2013 & $16.8 \%$ & $15.8 \%$ & $15.1 \%$ & $14.6 \%$ & $15.6 \%$ & $14.8 \%$ & $15.4 \%$ \\
\hline 2014 & $13.0 \%$ & $13.4 \%$ & $12.3 \%$ & $13.4 \%$ & $13.5 \%$ & $12.5 \%$ & $13.3 \%$ \\
\hline 2015 & $16.9 \%$ & $16.0 \%$ & $14.5 \%$ & $19.1 \%$ & $15.8 \%$ & $16.9 \%$ & $16.2 \%$ \\
\hline 2016 & $3.3 \%$ & $12.2 \%$ & $12.4 \%$ & $15.1 \%$ & $11.3 \%$ & $13.5 \%$ & $11.9 \%$ \\
\hline $\begin{array}{l}\text { Quan-Charlson Comorbidity } \\
\text { Index }{ }^{\mathrm{b}} \text {, mean } \pm \text { SD }\end{array}$ & $0.5 \pm 1.1$ & $1.2 \pm 1.5$ & $1.9 \pm 1.4$ & $0.9 \pm 1.3$ & $0.7 \pm 1.2$ & $0.9 \pm 1.3$ & $0.7 \pm 1.3$ \\
\hline Extra-articular manifestations ${ }^{\mathrm{b}, \mathrm{c}}, \%$ & $12.4 \%$ & $43.5 \%$ & $27.3 \%$ & $100.0 \%$ & $100.0 \%$ & $32.5 \%$ & $84.8 \%$ \\
\hline Gastro-related conditions ${ }^{\mathrm{b}, \mathrm{d}}, \%$ & $13.6 \%$ & $18.8 \%$ & $22.1 \%$ & $19.5 \%$ & $15.3 \%$ & $22.5 \%$ & $16.7 \%$ \\
\hline \multicolumn{8}{|l|}{ Drug use $\mathrm{b}^{\mathrm{b}}, \%$} \\
\hline NSAIDS & $17.0 \%$ & $33.3 \%$ & $43.7 \%$ & $42.5 \%$ & $20.5 \%$ & $51.5 \%$ & $28.3 \%$ \\
\hline Corticosteroids & $19.5 \%$ & $45.3 \%$ & $62.3 \%$ & $49.3 \%$ & $33.4 \%$ & $47.7 \%$ & $38.2 \%$ \\
\hline Biologics & $0.1 \%$ & $21.0 \%$ & $31.9 \%$ & $54.3 \%$ & $16.4 \%$ & $44.9 \%$ & $27.0 \%$ \\
\hline PDE4 inhibitors & $0.0 \%$ & $0.4 \%$ & $0.1 \%$ & $3.2 \%$ & $0.9 \%$ & $0.1 \%$ & $1.2 \%$ \\
\hline Opioids & $24.0 \%$ & $38.8 \%$ & $50.0 \%$ & $42.3 \%$ & $27.6 \%$ & $50.0 \%$ & $33.1 \%$ \\
\hline DMARDs & $1.5 \%$ & $37.6 \%$ & $67.1 \%$ & $56.4 \%$ & $17.4 \%$ & $39.4 \%$ & $28.8 \%$ \\
\hline
\end{tabular}

CID Chronic inflammatory disease, SD Standard deviation, HRU Healthcare resource utilization, RA Rheumatoid arthritis, PsA Psoriatic arthritis, PsO Psoriasis, AS Ankylosing spondylitis, NSAIDs Non-steroidal anti-inflammatory drugs, DMARDS Disease modifying anti-rheumatic drugs

${ }^{\text {a }}$ Measured at the index date.

${ }^{\mathrm{b}}$ Measured during the 12 -month baseline period.

'Extra-articular manifestations include cutaneous, ocular, cardiovascular, urogenital, pulmonary, and other manifestations such as enthesopathies, parapsoriasis, pityriasis, and other psoriasis and similar disorders.

${ }^{\mathrm{d} G a s t r o-r e l a t e d ~ c o n d i t i o n s ~ i n c l u d e ~ d i a r r h e a, ~ w e i g h t ~ l o s s, ~ b l o o d ~ i n ~ s t o o l, ~ a b d o m i n a l ~ p a i n, ~ g a s t r o i n t e s t i n a l ~ h e m o r r h a g e, ~ i s c h e m i c ~ c o l i t i s, ~ d y s p e p s i a, ~}$

and gastroenteritis.

\section{HRU}

Among patients with CIDs, patients who developed IBD had higher rates of hospital admissions (IRR $=2.91,95 \%$ $\mathrm{CI}=2.67-3.16, P<0.0001)$, days spent at the hospital (IRR $=3.46,95 \% \mathrm{CI}=3.04-3.91, P<0.0001)$, outpatient visits $(\mathrm{IRR}=1.35,95 \% \mathrm{CI}=1.31-1.40, P<0.0001)$, and ER visits $(\mathrm{IRR}=1.81,95 \% \mathrm{CI}=1.65-1.99, \quad P<0.0001)$ compared to those who did not develop IBD. Similarly, among condition-specific cohorts, those who developed IBD had higher rates of hospital admissions, days spent at the hospital, outpatient visits, and ER visits compared to patients who did not develop IBD (Fig. 4). In addition, among patients with CIDs, compared to those who did not develop IBD, patients who developed IBD were 


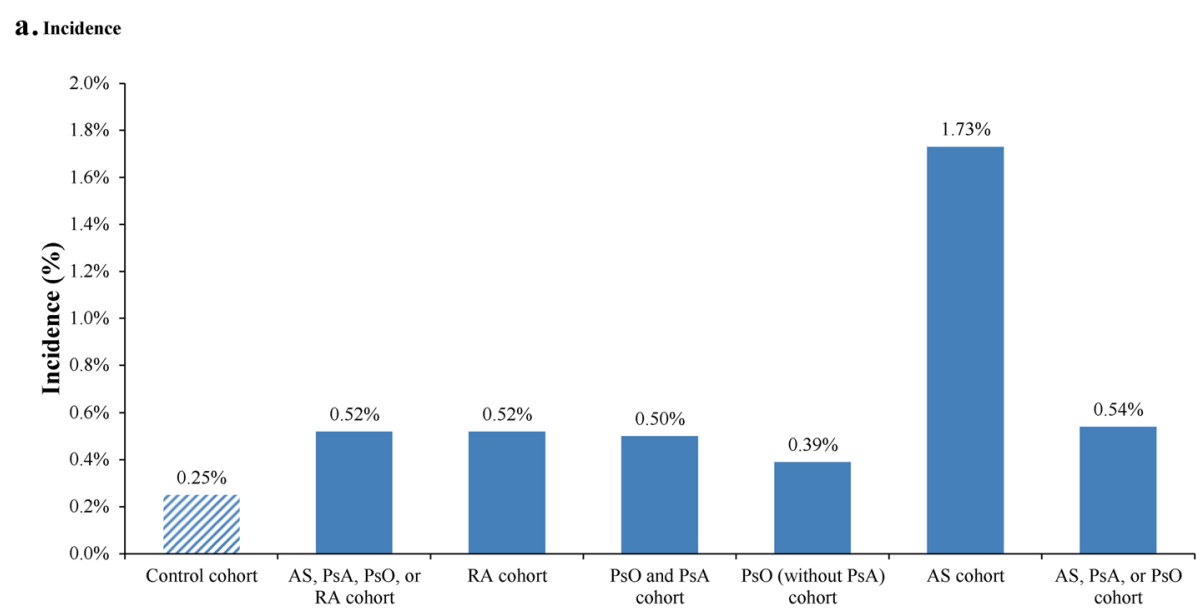

b. Prevalence

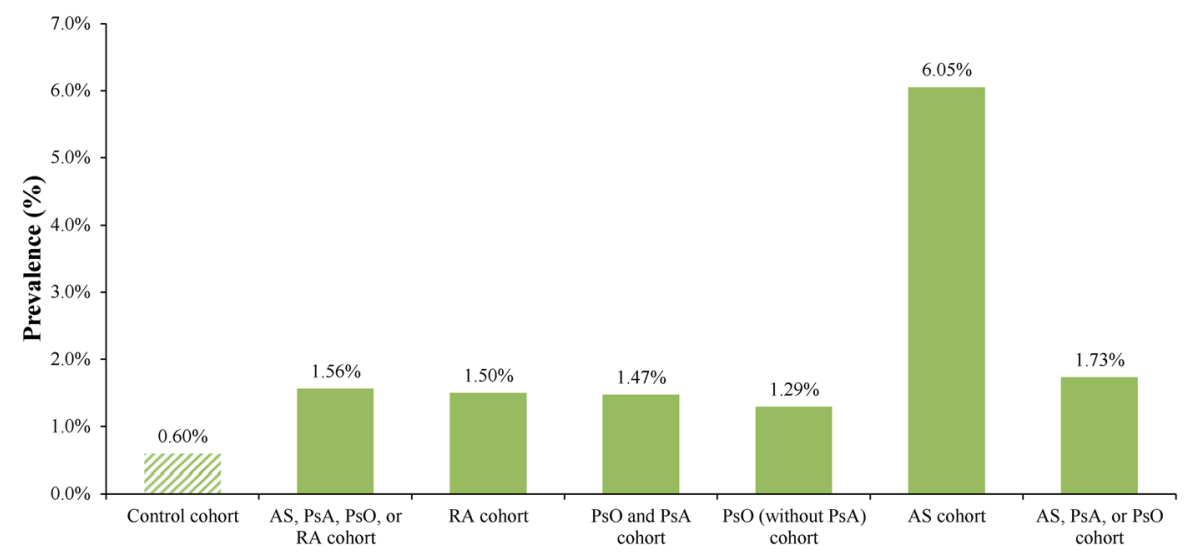

Fig. 3 One-year incidence rate (a) and prevalence (b) of IBD by type of CID. CID, chronic inflammatory disease; RA, rheumatoid arthritis; PsA, psoriatic arthritis; PsO, psoriasis; AS, ankylosing spondylitis; IBD, inflammatory bowel disease

more likely to have an IBD-related surgery (odds ratio $=11.7795 \% \mathrm{CI}=9.93-13.96$ ), such as bowel resection or pouch surgery/proctocolectomy with ileal pouch-anal anastomosis.

\section{Healthcare costs}

Among patients with CIDs, unadjusted mean all-cause total healthcare costs per year among those who developed IBD were $\$ 41,150$ versus $\$ 20,644$ among those who did not develop IBD. Similar trends were observed in condition-specific cohorts. In these cohorts, total costs ranged from $\$ 35,062$ (patients with $\mathrm{PsO}$ but without PsA) to $\$ 50,861$ (patients with RA) for patients with IBD and from $\$ 16,494$ (patients with $\mathrm{PsO}$ but without PsA) to $\$ 32,452$ (patients with PsO and PsA) for patients without IBD. The largest proportion of healthcare costs were attributed to medical costs (Table 2).

Compared to those who did not develop IBD, patients with CID who developed IBD had an average of $\$ 18,500$
(95\% $\mathrm{CI}=\$ 16,448-\$ 20,604 ; \quad P<0.0001) \quad$ higher total costs per year, including $\$ 15,121(95 \% \mathrm{CI}=\$ 13,015-\$ 17$, 164; $P<0.0001)$ higher medical costs and $\$ 3380$ (95\% $\mathrm{CI}=\$ 2712-\$ 4111 ; P<0.0001)$ higher pharmacy costs. The higher medical costs were driven by higher hospitalization costs (adjusted MYCD: \$8575, $P<$ 0.0001 ) and outpatient costs (adjusted MYCD: \$5544, $P<0.0001)$. The largest components of pharmacy cost among all CID patients were biologics (\$6706 versus \$4769; adjusted MYCD: \$1771, $P<0.0001)$ and DMARDs (\$4917 versus \$2047; adjusted MYCD: \$2751, $P<0.0001)$. Similar cost differences were observed among condition-specific cohorts. The total annual cost difference between CID with IBD and CID alone ranged from $\$ 14,922$ in the PsO and PsA cohort, to $\$ 21,792$ in the RA cohort; differences in medical costs ranged from $\$ 13,408$ in the PsO and PsA cohort to $\$ 19,033$ in the RA cohort, and differences in pharmacy costs ranged from $\$ 1514$ in the PsO and PsA cohort to \$3216 in the AS cohort (Fig. 5). 


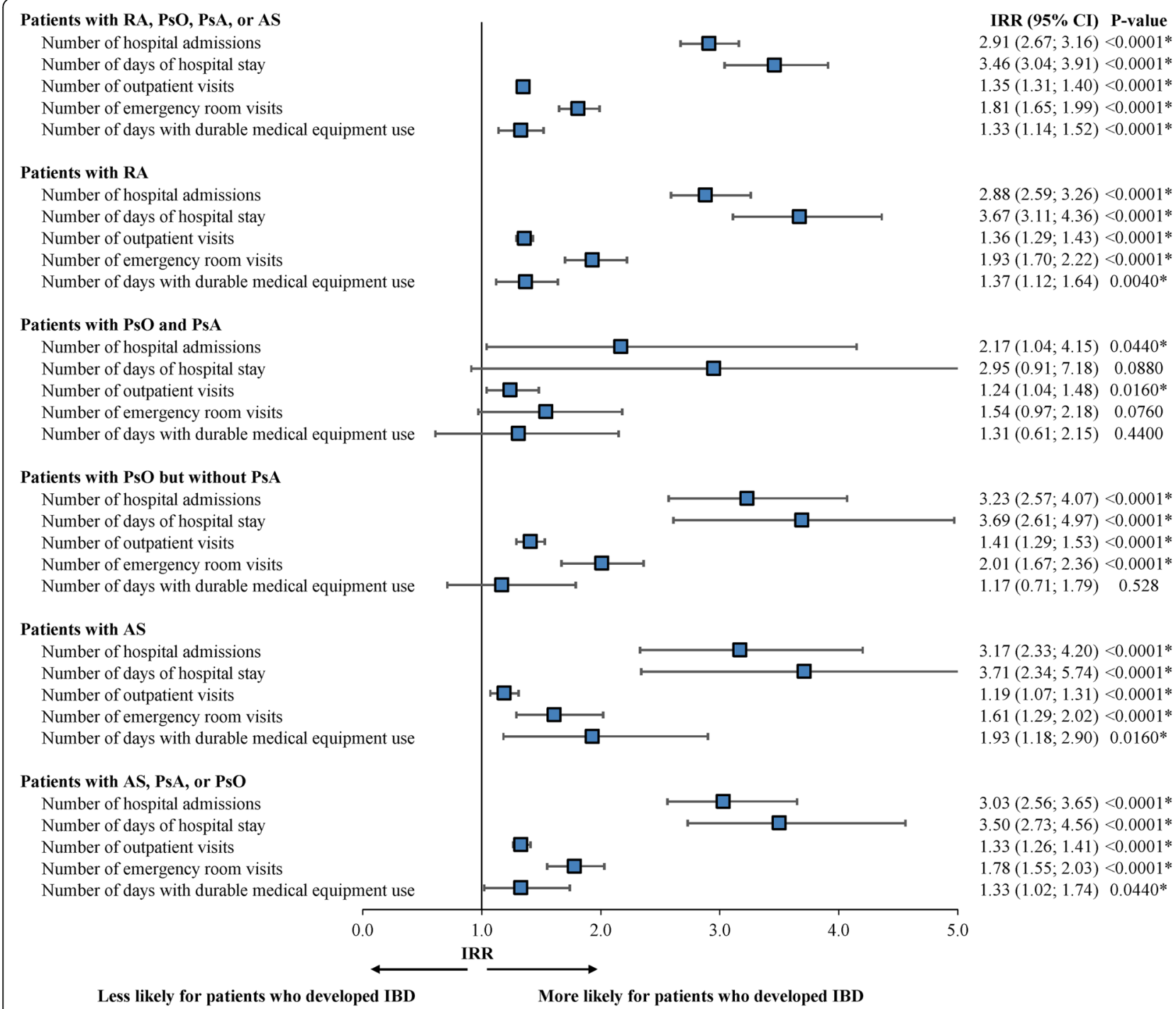

Fig. 4 Comparison of HRU during the 12-month observation period between patients developing versus not developing IBD. RA, rheumatoid arthritis; PsA, psoriatic arthritis; PsO, psoriasis; AS, ankylosing spondylitis; IBD, inflammatory bowel disease; HRU, healthcare resource utilization; IRR, incidence rate ratio; $\mathrm{Cl}$, confidence interval. * indicates that $p$-value $<0.05$

Compared to those who did not develop IBD, patients with CID who developed IBD incurred higher IBD surgery-related costs (\$1038 versus \$73; adjusted MYCD: $\$ 959, P<0.0001)$. Among the subset of CID patients who underwent IBD surgery, the cost of such a surgery was $>\$ 18,000$.

Among patients with CIDs who received DMARDs and/or corticosteroids, those who developed IBD $(N=$ 2020) incurred mean total all-cause healthcare costs of $\$ 44,192$ compared to $\$ 25,472$ for those who did not $(N=320,561)$, resulting in an adjusted MYCD of $\$ 17,605$ $(P<0.0001)$. Similar to the main analysis, this difference was largely driven by higher hospitalization costs (adjusted MYCD: \$7755), outpatient costs (adjusted MYCD:
\$5392), and pharmacy costs (adjusted MYCD: \$3612, all $P<0.0001)$.

\section{Discussion}

This study is among the first to provide estimates of real-world incidence and economic burden of IBD among patients with CIDs, overall, and separately in subsets of patients with RA, patients with PsO and PsA, patients with $\mathrm{PsO}$ but without PsA, patients with AS, and patients with AS, PsA, or PsO. Overall, the incidence of IBD among patients with CIDs was $0.52 \%$ and was $0.25 \%$ in the non-CID cohort. Patients with CIDs who developed IBD incurred on average \$14,922-\$21, 792 higher total healthcare costs and had higher HRU 
Table 2 Unadjusted costs by type of chronic inflammatory disease during the 12-month observation period

\section{All Patients: Patients with RA, PsO, PsA,
Total healthcare costs
Medical costs
Hospitalization costs
Outpatient costs
Emergency room costs
Durable medical equipment costs
Pharmacy costs}

\section{Patients with RA}

Total healthcare costs

Medical costs

Hospitalization costs

Outpatient costs

Emergency room costs

Durable medical equipment costs

Pharmacy costs

\section{Patients with PsO and PsA}

Total healthcare costs

Medical costs

Hospitalization costs

Outpatient costs

Emergency room costs

Durable medical equipment costs

Pharmacy costs

\section{Patients with PsO but without PsA}

Total healthcare costs

Medical costs

Hospitalization costs

Outpatient costs

Emergency room costs

Durable medical equipment costs

Pharmacy costs

\section{Patients with AS}

Total healthcare costs

Medical costs

Hospitalization costs

Outpatient costs

Emergency room costs

Durable medical equipment costs

Pharmacy costs

\section{Patients with AS, PsA, or PsO}

Total healthcare costs

Medical costs
Unadjusted all-cause costs per year, USD (2017), mean \pm SD [median]

Patients who developed IBD

Patients who did not develop IBD

$\boldsymbol{N}=\mathbf{2 7 7 8}$

$N=534,672$

$41,150 \pm 62,218[23,482]$

$29,735 \pm 59,281[10,781]$

$13,163 \pm 48,359[0]$

$14,595 \pm 25,168$ [6399]

$1836 \pm 5954$ [0]

$142 \pm 716[0]$

$11,414 \pm 18,916$ [3917]

\section{$N=1082$}

$50,861 \pm 71,823[32,696]$

$38,597 \pm 69,336[15,748]$

$17,888 \pm 58,399[0]$

$18,319 \pm 26,492$ [8400]

$2200 \pm 7188[0]$

$189 \pm 894$ [0]

$12,264 \pm 20,941$ [4715]

$$
N=106
$$

$50,162 \pm 57,420[35,973]$

$30,747 \pm 54,422[11,781]$

$9988 \pm 42,153[0]$

$19,042 \pm 25,040$ [7382]

$1581 \pm 4740[0]$

$136 \pm 517[0]$

$19,415 \pm 22,960[10,857]$

$$
\mathbf{N}=\mathbf{4 9 0}
$$

$35,062 \pm 56,249[17,763]$

$24,355 \pm 52,492[8468]$

$10,940 \pm 42,213[0]$

$11,646 \pm 23,071$ [5330]

$1665 \pm 4635[0]$

$104 \pm 564[0]$

$10,708 \pm 16,664$ [3533]

$$
\mathbf{N}=\mathbf{2 7 7}
$$

$47,410 \pm 53,738[34,535]$

$31,894 \pm 53,244[12,097]$

$12,165 \pm 42,744[0]$

$17,493 \pm 27,693$ [6757]

$2089 \pm 6233[0]$

$147 \pm 661[0]$

$15,516 \pm 18,356$ [9058]

$$
\mathbf{N}=\mathbf{9 8 2}
$$

$42,074 \pm 60,394[25,759]$

$28,425 \pm 57,753[10,267]$
$20,644 \pm 39,510$ [7867]

$13,004 \pm 35,906$ [3051]

$4059 \pm 23,848[0]$

$8063 \pm 21,660$ [2426]

$771 \pm 3706[0]$

$112 \pm 762[0]$

$7640 \pm 15,353$ [1462]

$N=205,178$

$27,026 \pm 43,583[14,242]$

$17,820 \pm 40,349$ [4836]

$5530 \pm 26,284$ [0]

$11,195 \pm 24,592$ [3651]

$950 \pm 4371$ [0]

$144 \pm 825[0]$

$9206 \pm 16,335$ [2184]

$N=21,144$

$32,452 \pm 38,332[24,341]$

$14,702 \pm 32,814$ [3508]

$3607 \pm 19,405[0]$

$10,236 \pm 22,143$ [2843]

$755 \pm 3772$ [0]

$17,750 \pm 21,576[8425]$

$N=124,460$

$16,494 \pm 36,155$ [5807]

$8902 \pm 32,175$ [2067]

$2746 \pm 22,967[0]$

$5498 \pm 18,670$ [1681]

$568 \pm 2858$ [0]

$89 \pm 641[0]$

$7592 \pm 15,405$ [1326]

$N=15,752$

$28,325 \pm 41,809[17,443]$

$16,045 \pm 37,433$ [3961]

$4242 \pm 22,895[0]$

$10,861 \pm 24,478[3162]$

$819 \pm 3427$ [0]

$123 \pm 944[0]$

$12,280 \pm 19,354$ [3021]

$N=182,336$

$20,734 \pm 37,661$ [8332]

$10,858 \pm 33,031$ [2443]
$104 \pm 763[0]$ 
Table 2 Unadjusted costs by type of chronic inflammatory disease during the 12-month observation period (Continued)

\begin{tabular}{lcc}
\hline & \multicolumn{1}{c}{ Unadjusted all-cause costs per year, USD (2017), mean \pm SD [median] } \\
\cline { 2 - 3 } & Patients who developed IBD & Patients who did not develop IBD \\
\hline Hospitalization costs & $11,719 \pm 47,236[0]$ & $7021 \pm 22,291[0]$ \\
Outpatient costs & $14,843 \pm 25,322[6313]$ & $628 \pm 3091[0]$ \\
Emergency room costs & $1747 \pm 4986[0]$ & $95 \pm 684[0]$ \\
Durable medical equipment costs & $116 \pm 565[0]$ & $9877 \pm 17,339[1846]$ \\
Pharmacy costs & $13,649 \pm 18,296[5311]$ & \\
\hline
\end{tabular}

RA Rheumatoid arthritis, PSA Psoriatic arthritis, PsO Psoriasis, AS Ankylosing spondylitis, IBD Inflammatory bowel disease, USD US dollars

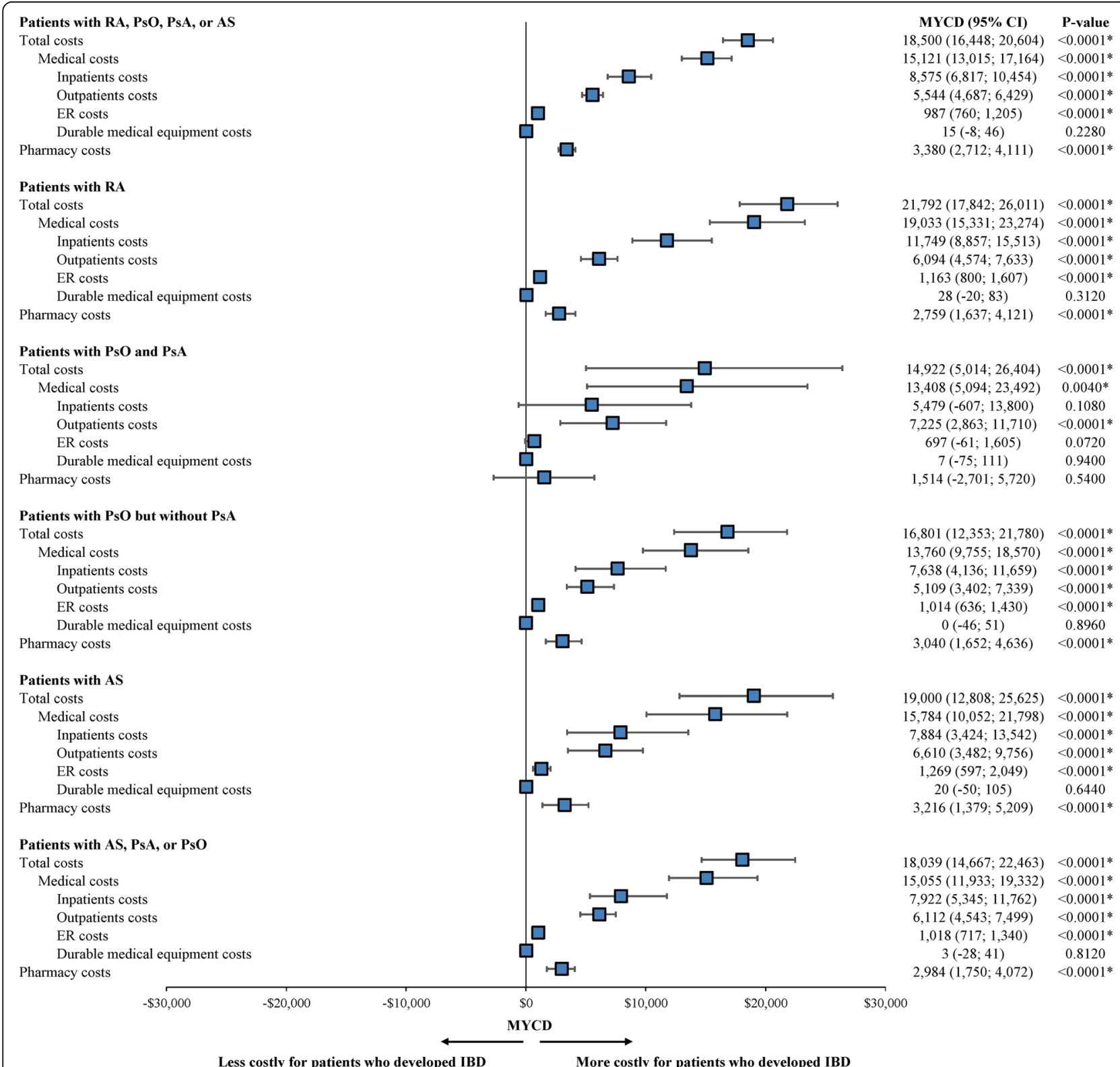

Fig. 5 Comparison of costs during the 12-month observation period between patients developing versus not developing IBD. RA, rheumatoid arthritis; PsA, psoriatic arthritis; PsO, psoriasis; AS, ankylosing spondylitis; IBD, inflammatory bowel disease; MYCD, mean yearly cost difference; Cl, confidence interval. ${ }^{*}$ indicates that $p$-value $<0.05$ 
per year, including hospital admissions, days spent at the hospital, outpatient visits, and ER visits compared to patients without IBD. Important drivers of the cost difference included hospitalization costs, outpatient costs, and costs related to the use of biologics and DMARDs. Another possible driver could be costs related to endoscopy/colonoscopy procedures, which were captured as part of all-cause healthcare costs, but were not reported separately.

The one-year incidence of IBD varied among subsets of patients with different CIDs, ranging from 0.39\% in patients with $\mathrm{PsO}$ but without PsA to $1.73 \%$ among patients with AS. The one-year prevalence of IBD was higher across all cohorts, but consistent trends were observed, with values ranging from $1.29 \%$ in patients with PsO but without PsA to $6.05 \%$ in patients with AS. A previous study based on similar data reported IBD incidence of $2.5 \%$ in patients with PsA and $4.1 \%$ in patients with AS during the one-year period following the initial claim for PsA/AS [28]. IRs were higher because the definition of IBD included Crohn's disease, ulcerative colitis, and gastrointestinal disturbances (i.e., gastroenteritis, colitis, and gastritis) as opposed to only Crohn's disease and ulcerative colitis in the current study. Although crude IRs vary between previous estimates and the present study, both suggest a higher rate of IBD among patients with AS compared to other CID types. In a systematic review of 156 studies, the pooled prevalence of IBD among patients with AS was 6.8\% [30], consistent with the one-year prevalence of IBD among patients with AS reported in the current study (6.05\%). In addition, the IR of IBD in patients with PsO and PsA was higher than in patients with $\mathrm{PsO}$ without PsA. This result is consistent with the literature, as a previous study showed that patients with concomitant $\mathrm{PsO}$ and PsA were almost twice as likely to develop IBD as patients with $\mathrm{PsO}$ only [31]. Having PsA in addition to $\mathrm{PsO}$ may also explain why the observed IR of IBD was more similar between the RA cohort and the PsO with PsA cohort than between the RA cohort and the PsO without PsA cohort.

While it was anticipated that patients with IBD and CID would incur higher costs than patients with CID alone, this study is the first to quantify the incremental costs and HRU associated with IBD among patients with AS, PsA, PsO, or RA, stratified by type of CID. Of note, the difference in total all-cause healthcare costs found in our study $(\$ 18,500)$ is similar to that observed in recent analyses which assessed the incremental burden of patients with IBD versus non-IBD controls (IBD: \$16,031 [27], CD only: $\$ 17,463$ [32], UC only: $\$ 11,029$ [33]). Although comparisons with results from other analyses are prone to confounding, this suggests that the incremental burden associated with IBD in a population of patients with CID is similar to that observed in the general population.

Only one previous analysis from Bergman et al. compared HRU and costs between patients with CID who subsequently developed IBD and controls who did not develop IBD [28]. However, this study only included patients with PsA or AS, thereby precluding the generalization of findings to other CIDs. Indeed, CIDs show substantial heterogeneity in their incidence, prevalence, and the characteristics of affected patients (i.e., age, gender, associated comorbidities, etc.) [34], which may impact HRU and costs. For this reason, all patients with CIDs were included in the present study and were classified into different cohorts based on the type of CID(s) diagnosis.

The total annual direct costs of all patients with IBD in the US in 2014 was estimated to be between $\$ 11$ billion and $\$ 28$ billion [35]. With the rising prevalence of both Crohn's disease and ulcerative colitis, IBD represents the leading chronic gastrointestinal disease with increasing healthcare expenditures in the US [36]. There has been a shift in IBD costs from inpatient to outpatient care since the introduction of biologic therapies as the standard of care [36]. Biologic agents are increasingly used to treat both CID and IBD when conventional therapies fail, such as in CID patients unresponsive or intolerant to NSAIDs [37]. While some biologics are approved for the treatment of IBD (i.e., certolizumab pegol, subcutaneous golimumab, adalimumab, infliximab, vedolizumab, and natalizumab), biologic therapies with certain mechanisms of action, such as IL-17 antagonists and etanercept, have been linked with an increased risk of new onset IBD or exacerbation of existing IBD. A number of studies have noted that patients with Crohn's disease experienced exacerbation of their symptoms after receiving treatment with secukinumab [14] or brodalumab [15]. Evidence from clinical trials has also shown higher incidence of IBD in patients treated with different IL-17 antagonists [14, 16, 17]. Similarly, a large realworld retrospective longitudinal cohort study of the incidence of IBD among patients with CIDs treated with IL-17 blockers or PDE4 inhibitors found that treatment with IL-17 antagonists was associated with more than 3fold higher odds of having IBD relative to patients naïve to biologics and patients treated with biologics not indicated for the treatment of IBD [38]. Finally, a number of studies also found that etanercept was associated with exacerbation of existing IBD or new onset IBD $[21,22]$.

Even if the incidence of IBD remains low (as was shown in the previous studies and by the results of the current study), the occurrence of concomitant IBD in a few cases was shown to be associated with a considerable increase in total healthcare costs for patients with CIDs. It may also result in adverse outcomes [39], 
especially in patients who already have difficult chronic conditions such as AS, PsA, PsO, or RA. The findings of the present study suggest that treatment decisions should consider the increased risk and associated HRU and healthcare costs of IBD. Healthcare providers should be aware of the cost burden of IBD and treatments that optimize cost-efficiency when treating patients with CID.

The high rates of opioid use observed in the current study reflect both the pain burden associated with CIDs as well as US-specific circumstances. Overall, $38.8 \%$ of patients with CIDs and $24.0 \%$ of those without CIDs exhibited opioid use at baseline. This difference is likely driven by patients who were prescribed opioids to manage the pain associated with CIDs. Patients with CIDs are known to exhibit higher use of prescription opioids than the general population for pain management [40, 41]. A study by Zhdanava et al. that included patients with $\mathrm{PsO}$ reported rates of opioid use that were consistent with those of the present study ( $\mathrm{PsO}$ cohort: 42.8\%, matched non-PsO cohort: 30.7\%) [41]. In absolute terms, the rate of opioid use in the non-CID cohort of our study (24.0\%) was nonetheless high, and that reported by Zhdanava et al. (30.7\%) was in the same range. This is likely due to the marked increase of opioid use across the US over the past few years [42].

The strengths of the study include its large sample size and estimation of incidence, HRU, and healthcare costs and its components in an overall sample of patients with CIDs as well as among subsets of patients with specific CIDs, which has never been published before. The study findings should also be interpreted in the context of its limitations. First, claims databases may contain inaccuracies from missing data and occasional coding errors. Second, as with all observational studies, results from the current study may be affected by residual confounding from measured and unmeasured factors. In particular, since incidence rates of IBD were only reported descriptively for each patient cohort, confounding related to differences in patients' baseline characteristics (such as age) may remain. Third, the data used in this study covered only commercially-insured patients who may have a higher socioeconomic status and may not be representative of the general US population. For example, uninsured individuals and Medicaid beneficiaries were not included in the current study. Fourth, when describing the one-year IR of IBD, no adjustments for potential confounders were made to account for differences in characteristics between the cohorts; therefore, no statistical comparisons between cohorts were conducted for IRs. Finally, patients may have had PsA for many years. Therefore, the number of patients with $\mathrm{PsO}$ and PsA may have been underestimated in the current study among patients with PsA, given that there may have been no claim for $\mathrm{PsO}$ among patients with PsA in recent years.

\section{Conclusions}

In patients with CIDs, developing IBD was associated with substantially higher HRU and healthcare costs compared to patients not developing IBD. Treatment decisions for patients with CIDs should factor in the risk of developing of IBD as well. Such considerations have the potential to improve cost-effectiveness and health outcomes for patients with CIDs.

\section{Abbreviations \\ AS: Ankylosing spondylitis; Cl: Confidence interval; CID: Chronic inflammatory disease; DMARD: Disease-modifying anti-rheumatic drug; EAM: Extra-articular manifestations; ER: Emergency room; GLM: Generalized linear model; HRU: Healthcare resource utilization; IBD: Inflammatory bowel disease; ICD-10 CM: International Classification of Diseases, Tenth Revision, Clinical Modification; ICD-9 CM: International Classification of Diseases, Ninth Revision, Clinical Modification; IL-17: Interleukin-17; IR: Incidence rate; IRR: Incidence rate ratios; MYCD: Mean yearly cost difference; NSAID: Non-steroidal anti-inflammatory drug; PDE4: Phosphodiesterase 4; PPPY: Per-patient-per-year; PsA: Psoriatic arthritis; PsO: Psoriasis; \\ Quan-CCl: Quan-Charlson Comorbidity Index; RA: Rheumatoid arthritis; SD: Standard deviation; US: United States}

\section{Acknowledgements}

Not applicable.

\section{Authors' contributions}

$\mathrm{DPH}, \mathrm{SDC}, \mathrm{LAE}, \mathrm{KS}$, and JUS made substantial contributions to the study conception and design, as well as the interpretation of data; were involved in drafting the manuscript and revising it critically for important intellectual content; and gave final approval of the version to be published. BE and PL made substantial contributions to the study conception and design, the acquisition, collection, analysis, and interpretation of the data; were involved in drafting the manuscript and revising it critically for important intellectual content; and gave final approval of the version to be published. All authors participated sufficiently in the work to take public responsibility for appropriate portions of the content and agreed to be accountable for all aspects of the work by ensuring that questions related to the accuracy or integrity of any part of the work are appropriately investigated and resolved. All authors read and approved the final version of the manuscript for submission.

\section{Funding}

This research was funded by Janssen Scientific Affairs, LLC. The sponsor had a role in the design of the study, in the collection, analysis, and interpretation of data, and in writing the manuscript.

\section{Availability of data and materials}

The data that support the findings of this study are available from IBM but restrictions apply to the availability of these data, which were used pursuant to a data use agreement. The data are available through requests made directly to IBM, subject to IBM's requirements for data access.

\section{Ethics approval and consent to participate}

As this was an analysis of claims data, IRB approval was not required. Per Title 45 of CFR, Part 46.101(b) (4) (www.hhs.gov/ohrp/humansubjects/ guidance/45cfr46.html\#46.101), the administrative claims data analysis of our study is exempt from the IRB review for two reasons: (1) it is a retrospective analysis of existing data (hence no patient intervention or interaction), (2) no patient-identifiable information is included in the claims dataset.

Consent for publication

Not applicable. 


\section{Competing interests}

$\mathrm{BE}$ and $\mathrm{PL}$ are employees of Analysis Group, Inc., a consulting company which provided paid consulting services to Janssen Scientific Affairs, LLC for the conduct of this study. SDC, LAE, and KS are employees of Janssen Scientific Affairs, LLC and shareholders of Johnson \& Johnson, of which Janssen Scientific Affairs, LLC is a wholly-owned subsidiary. DPH reports the following affiliations: Janssen (consulting), Takeda (consulting), AbbVie (consulting), Salix (consulting), and Pfizer (consulting and research support). JUS reports the following affiliations: Janssen (consulting), UCB (consulting), Novartis (consulting and research support), Amgen (consulting and research support [via NIH Target]), and Pfizer (research support).

\section{Author details}

'NYU Langone Health, 240 East 38th Street, 23rd Floor, New York, NY 10016, USA. ${ }^{2}$ Janssen Scientific Affairs, LLC, 800 Ridgeview Drive, Horsham, PA 19044, USA. ${ }^{3}$ Drexel University College of Medicine, Philadelphia, PA, USA.

${ }^{4}$ Analysis Group, Inc., Montréal, Québec, Canada.

\section{Received: 7 August 2019 Accepted: 16 January 2020}

\section{Published online: 02 April 2020}

\section{References}

1. Bernstein CN, Wajda A, Blanchard JF. The clustering of other chronic inflammatory diseases in inflammatory bowel disease: a population-based study. Gastroenterology. 2005;129(3):827-36.

2. Cohen AD, Dreiher J, Birkenfeld S. Psoriasis associated with ulcerative colitis and Crohn's disease. J Eur Acad Dermatol Venereol. 2009;23(5):561-5.

3. Rudwaleit M, Baeten D. Ankylosing spondylitis and bowel disease. Best Pract Res Clin Rheumatol. 2006;20(3):451-71.

4. Skroza N, Proietti I, Pampena R, et al. Correlations between psoriasis and inflammatory bowel diseases. Biomed Res Int. 2013;2013:983902.

5. Cotsapas C, Voight BF, Rossin E, et al. Pervasive sharing of genetic effects in autoimmune disease. PLoS Genet. 2011;7(8):e1002254.

6. Fiorino G, Omodei PD. Psoriasis and inflammatory bowel disease: two sides of the same coin? J Crohns Colitis. 2015;9(9):697-8.

7. Monteleone I, Pallone F, Monteleone G. Th17-related cytokines: new players in the control of chronic intestinal inflammation. BMC Med. 2011;9:122.

8. Baliwag J, Barnes DH, Johnston A. Cytokines in psoriasis. Cytokine. 2015; 73(2):342-50.

9. Bui VL, Brahn E. Cytokine targeting in rheumatoid arthritis. Clin Immunol. 2018;206:3-8.

10. Neurath MF. Cytokines in inflammatory bowel disease. Nat Rev Immunol. 2014;14(5):329-42.

11. Kornbluth A, Sachar DB. Practice parameters Committee of the American College of G. ulcerative colitis practice guidelines in adults: American college of gastroenterology, practice parameters committee. Am J Gastroenterol. 2010;105(3):501-23 quiz 524.

12. Lichtenstein GR, Loftus EV, Isaacs KL, Regueiro MD, Gerson LB, Sands BE. ACG clinical guideline: Management of Crohn's disease in adults. Am J Gastroenterol. 2018;113(4):481-517.

13. Ward MM, Deodhar A, Akl EA, et al. American College of Rheumatology/spondylitis Association of America/Spondyloarthritis research and treatment network 2015 recommendations for the treatment of Ankylosing spondylitis and nonradiographic axial Spondyloarthritis. Arthritis Rheumatol. 2016;68(2):282-98.

14. Hueber W, Sands BE, Lewitzky S, et al. Secukinumab, a human anti-IL-17A monoclonal antibody, for moderate to severe Crohn's disease: unexpected results of a randomised, double-blind placebo-controlled trial. Gut. 2012; 61(12):1693-700.

15. Targan SR, Feagan BG, Vermeire S, et al. Mo2083: a randomized, doubleblind, placebo-controlled study to evaluate the safety, tolerability, and efficacy of AMG 827 in subjects with moderate to severe Crohn's disease. Gastroenterology. 2012;143:e26.

16. Baeten D, Sieper J, Braun J, et al. Secukinumab, an interleukin-17A inhibitor, in Ankylosing spondylitis. N Engl J Med. 2015;373(26):2534-48.

17. Gordon KB, Blauvelt A, Papp KA, et al. Phase 3 trials of Ixekizumab in moderate-to-severe plaque psoriasis. N Engl J Med. 2016;375(4):345-56.

18. US Food and Drug Administration. Highlights of Precribing Information - CONSENTYX (secukinumab). 2018; Available from: https://www. accessdata.fda.gov/drugsatfda_docs/label/2018/125504s013lbl.pdf. Accessed 27 Feb 2018.
19. US Food and Drug Administration. Highlights of Precribing Information TALTZ (ixekizumab). 2018; Available from: https://www.accessdata.fda.gov/ drugsatfda_docs/label/2018/125521Orig1 s002lbl.pdf. Accessed 16 May 2018.

20. US Food and Drug Administration. Highlights of Precribing Information SILIQ (brodalumab). 2018; Available from: https://www.accessdata.fda.gov/ drugsatfda_docs/label/2018/125521Orig1s002lbl.pdf. Accessed 16 May 2018.

21. O'Toole A, Lucci M, Korzenik J. Inflammatory bowel disease provoked by Etanercept: report of 443 possible cases combined from an IBD referral center and the FDA. Dig Dis Sci. 2016;61(6):1772-4.

22. Zhou W, Huang Y, Lai J, Lu J, Feely M, Liu X. Anti-inflammatory biologics and anti-Tumoral immune therapies-associated colitis: a focused review of literature. Gastroenterol Res. 2018;11(3):174-88.

23. Bounthavong M, Li M, Watanabe $\mathrm{JH}$. An evaluation of health care expenditures in Crohn's disease using the United States medical expenditure panel survey from 2003 to 2013. Res Social Adm Pharm. 2017;13(3):530-8.

24. Gibson TB, Ng E, Ozminkowski RJ, et al. The direct and indirect cost burden of Crohn's disease and ulcerative colitis. J Occup Environ Med. 2008;50(11):1261-72.

25. Gunnarsson C, Chen J, Rizzo JA, Ladapo JA, Lofland JH. Direct health care insurer and out-of-pocket expenditures of inflammatory bowel disease: evidence from a US national survey. Dig Dis Sci. 2012;57(12):3080-91.

26. Kappelman MD, Rifas-Shiman SL, Porter CQ, et al. Direct health care costs of Crohn's disease and ulcerative colitis in US children and adults. Gastroenterology. 2008;135(6):1907-13.

27. Park KT, Ehrlich OG, Allen Jl, et al. The Cost of Inflammatory Bowel Disease: An Initiative From the Crohn's \& Colitis Foundation. Inflamm Bowel Dis. 2019;26(1):1-10.

28. Bergman MJ, Zueger P, Song J, Pivneva I, Betts KA, Joshi AD. Inflammatory bowel disease is associated with a substantial economic burden in patients with psoriatic arthritis and in patients with ankylosing spondylitis. Chicago: American College of Rheumatology Annual Meeting; 2018.

29. Quan H, Li B, Couris CM, et al. Updating and validating the Charlson comorbidity index and score for risk adjustment in hospital discharge abstracts using data from 6 countries. Am J Epidemiol. 2011;173(6):676-82.

30. Stolwijk C, van Tubergen A, Castillo-Ortiz JD, Boonen A. Prevalence of extraarticular manifestations in patients with ankylosing spondylitis: a systematic review and meta-analysis. Ann Rheum Dis. 2015;74(1):65-73.

31. Li WQ, Han JL, Chan AT, Qureshi AA. Psoriasis, psoriatic arthritis and increased risk of incident Crohn's disease in US women. Ann Rheum Dis. 2013;72(7):1200-5.

32. Pilon D, Obando C, Ding Z, et al. K3: Direct and Indirect Costs Associated with Crohn's Disease in the United States. J Manag Care Spec Pharm. 2019; 25(10-a Suppl):S72-3.

33. Pilon D, Obando C, Ding Z, et al. Su1781 - The Economic Burden of Ulcerative Colitis in the United States. Gastroenterology. 2019;156(6):S-609.

34. El-Gabalawy H, Guenther LC, Bernstein CN. Epidemiology of immunemediated inflammatory diseases: incidence, prevalence, natural history, and comorbidities. J Rheumatol Suppl. 2010;85:2-10.

35. Crohn's and Colitis Foundation of America. The Facts about Inflammatory Bowel Disease. 2014; http://www.crohnscolitisfoundation.org/assets/pdfs/ updatedibdfactbook.pdf.

36. Park KT, Bass D. Inflammatory bowel disease-attributable costs and costeffective strategies in the United States: a review. Inflamm Bowel Dis. 2011; 17(7):1603-9.

37. Matsuoka K, Kobayashi T, Ueno F, et al. Evidence-based clinical practice guidelines for inflammatory bowel disease. J Gastroenterol. 2018;53(3):305-53.

38. Emond B, Ellis L, Chakravarty SD, Ladouceur M, Lefebvre P. Real-world incidence of inflammatory bowel disease among patients with other chronic inflammatory diseases treated with interleukin-17a or phosphodiesterase 4 inhibitors. Curr Med Res Opin. 2019;35(10):1751-9.

39. Lonnfors $S$, Vermeire $S$, Greco M, Hommes D, Bell C, Avedano L. IBD and health-related quality of life -- discovering the true impact. J Crohns Colitis. 2014:8(10):1281-6.

40. Chen SK, Feldman CH, Brill G, Lee YC, Desai RJ, Kim SC. Use of prescription opioids among patients with rheumatic diseases compared to patients with hypertension in the USA: a retrospective cohort study. BMJ Open. 2019;9(6):e027495.

41. Zhdanava M, Teeple A, Pilon D, Shak N, Fitzgerald T, Lefebvre P. PSY10 opioid and healthcare resource use among privately insured moderate-tosevere psoriasis patients in the us. Value Health. 2019;22:S376. 
42. National Academies of Sciences Engineering and Medicine; Health and Medicine Division; Board on Health Sciences Policy; Committee on Pain Management and Regulatory Strategies to Address Prescription Opioid Abuse. Pain Management and the Opioid Epidemic: Balancing Societal and Individual Benefits and Risks of Prescription Opioid Use. Washington (DC): National Academies Press (US); 2017.

\section{Publisher's Note}

Springer Nature remains neutral with regard to jurisdictional claims in published maps and institutional affiliations.

- fast, convenient online submission

- thorough peer review by experienced researchers in your field

- rapid publication on acceptance

- support for research data, including large and complex data types

- gold Open Access which fosters wider collaboration and increased citations

- maximum visibility for your research: over $100 \mathrm{M}$ website views per year

At $\mathrm{BMC}$, research is always in progress. 